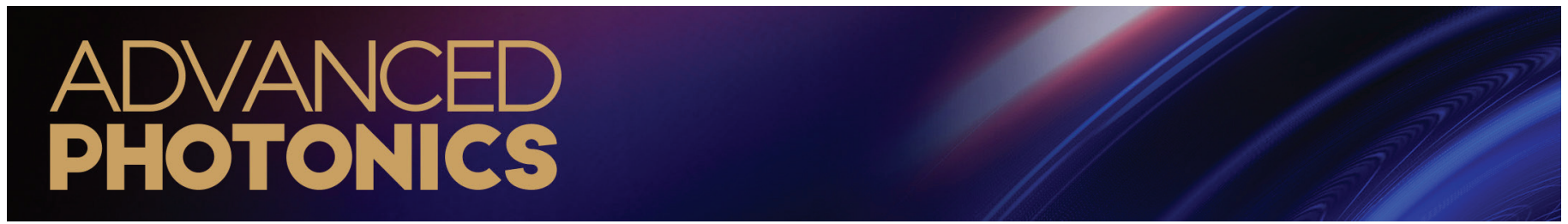

\title{
Near-perfect microlenses based on graphene microbubbles
}

\author{
Han Lin, ${ }^{\mathrm{a}, *}$ Scott Fraser, ${ }^{\mathrm{a}}$ Minghui Hong, ${ }^{\mathrm{b}}$ Manish Chhowalla, ${ }^{\mathrm{c}}$ Dan Li, ${ }^{\mathrm{d}, \mathrm{e}}$ and Baohua Jia ${ }^{\mathrm{a}, *}$ \\ a Swinburne University of Technology, Centre for Microphotonics, Faculty of Science, Engineering, and Technology, Hawthorn, Australia \\ ${ }^{b}$ National University of Singapore, Department of Electrical and Computer Engineering, Singapore \\ ${ }^{c}$ Rutgers University, Department of Materials Science and Engineering, Piscataway, New Jersey, United States \\ dUniversity of Melbourne, Department of Chemical Engineering, Melbourne, Australia \\ eMonash University, Monash Centre for Atomically Thin Materials, Department of Materials Science and Engineering, Clayton, Australia
}

\begin{abstract}
Microbubbles acting as lenses are interesting for optical and photonic applications such as volumetric displays, optical resonators, integration of photonic components onto chips, high-resolution spectroscopy, lithography, and imaging. However, stable, rationally designed, and uniform microbubbles on substrates such as silicon chips are challenging because of the random nature of microbubble formation. We describe the fabrication of elastic microbubbles with a precise control of volume and curvature based on femtosecond laser irradiated graphene oxide. We demonstrate that the graphene microbubbles possess a near-perfect curvature that allows them to function as reflective microlenses for focusing broadband white light into an ultrahigh aspect ratio diffraction-limited photonic jet without chromatic aberration. Our results provide a pathway for integration of graphene microbubbles as lenses for nanophotonic components for miniaturized lab-on-a-chip devices along with applications in high-resolution spectroscopy and imaging.
\end{abstract}

Keywords: graphene microbubble; photonic jet; microlens.

Received Jun. 21, 2020; revised manuscript received Aug. 24, 2020; accepted for publication Sep. 8, 2020; published online Oct. 7, 2020.

(C) The Authors. Published by SPIE and CLP under a Creative Commons Attribution 4.0 Unported License. Distribution or reproduction of this work in whole or in part requires full attribution of the original publication, including its DOI.

[DOI: 10.1117/1.AP.2.5.055001]

\section{Introduction}

Microbubbles are widely applied as actuators in microfluidic devices $^{1}$ for microfluidic mixing, ${ }^{2}$ ink-jet printing, ${ }^{3}$ and logic circuitry. ${ }^{4}$ More importantly, microbubbles have demonstrated broad applications in therapeutic and medical imaging, ${ }^{5}$ biomedicine, ${ }^{6}$ and cell and DNA trapping and manipulation. ${ }^{7}$ In addition, applications of microbubbles in photonics for lithography $^{8}$ and optical resonators have recently been demonstrated. ${ }^{9}$ For applications in imaging, trapping, and photonics, creation of bubbles at accurate positions with a controllable volume, curvature, and stability is essential. However, currently microbubbles can be generated with ultrasonic waves or laser pulses only when substrates are immersed in liquids, ${ }^{10}$ which are unstable and occur at random locations. For integration into biological or photonic applications, the generation of well controlled and stable microbubbles fabricated using a technique that is compatible

*Address all correspondence to Han Lin, E-mail: hanlin@swin.edu.au; Baohua Jia, E-mail: bjia@swin.edu.au with current processing technologies is highly desirable but has not yet been demonstrated. In this paper, we demonstrate the generation of microbubbles on the surface of a solid material using femtosecond laser pulse induced gas generation in a layer of graphene material for the first time. The position, volume, and curvature of the microbubbles are precisely controlled. Such a high-quality bubble can be used for advanced optoelectronic devices with high-precision requirements. As a showcase, nearperfect lenses for focusing broadband white light into an ultrahigh aspect ratio diffraction-limited photonic jet without chromatic aberration are demonstrated in this paper. Our results provide a pathway for integration of graphene microbubbles as dynamic and high-precision nanophotonic components for miniaturized lab-on-a-chip devices, along with applications in high-resolution spectroscopy and medical imaging.

\section{Materials and Methods}

The impermeability of graphene has been used to generate random microbubbles ${ }^{11}$ and tune their properties by electric fields. ${ }^{12}$ 


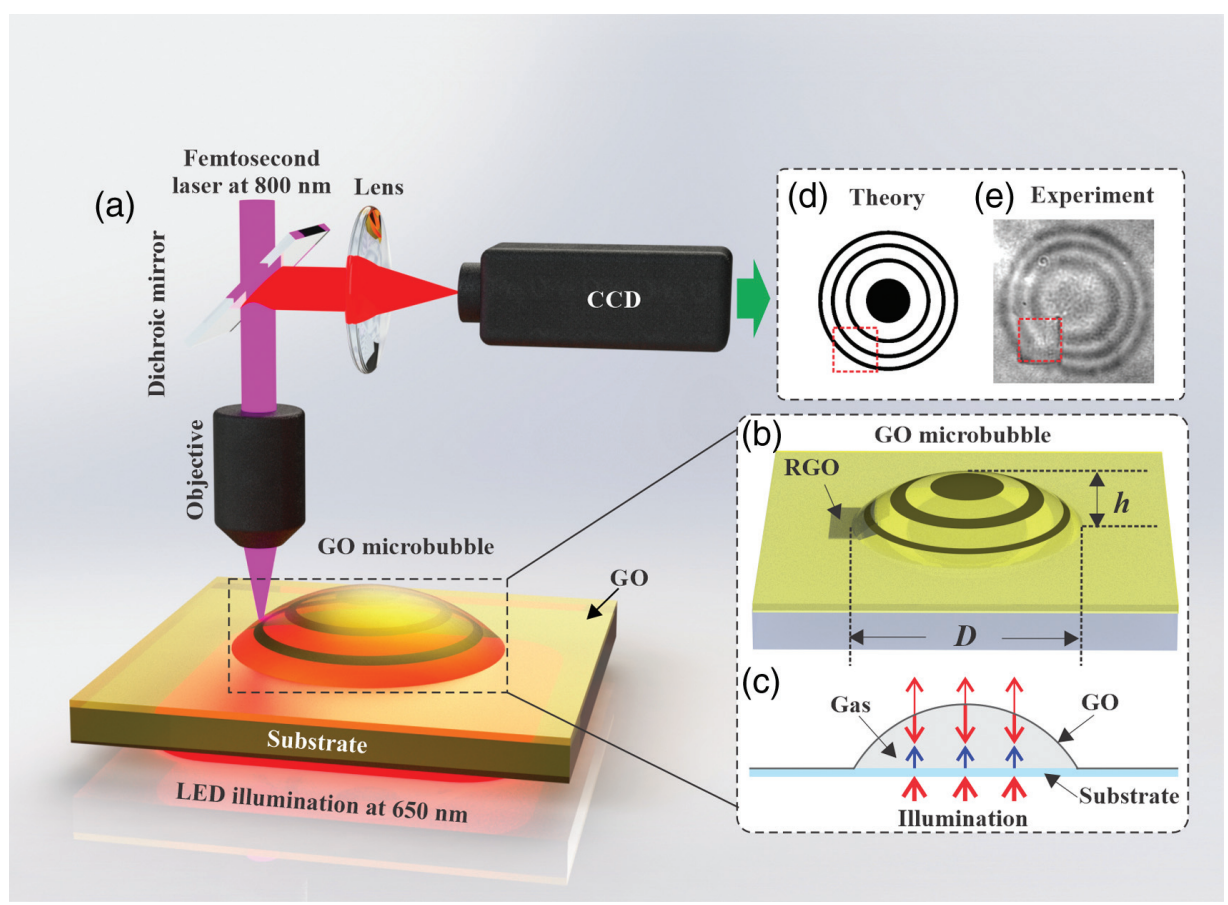

Fig. 1 On-demand generation and in situ characterization of the GO microbubbles. (a) Photoreduction setup with a femtosecond laser beam. The GO microbubbles are generated at the desired positions in an integrated platform. (b) Schematic of a GO microbubble. (c) The interference at the gas-bubble and gas-substrate interfaces. (d) Theoretical schematic of the Newton's rings that can be observed using a CCD camera. (e) Experimental microscopic image of the observed Newton's rings.

We irradiate graphene oxide $(\mathrm{GO})$ with a focused $\operatorname{laser}^{13-15}$ in precise locations using a commercial direct laser writing system (Innofocus NanoPrint ${ }^{3 \mathrm{D}}$ ) to create microbubbles in highly localized regions, as shown in Fig. 1(a). In this study, we use the low repetition rate $(1 \mathrm{kHz})$ femtosecond laser writing technique, which is considered to be a "cold" process, ${ }^{16}$ because the highinstant temperature achieved by a femtosecond laser pulse decreases to room temperature on the timescale of microseconds, before the irradiation of the next femtosecond pulse. Thus the GO film cannot be heated up by the femtosecond laser during the reduction process. The laser irradiation leads to a reduction of GO via evolution of oxygen functional groups. ${ }^{17,18}$ These evolved gaseous species ${ }^{19,20}$ are trapped within the reduced GO, forming near-perfect microbubbles at precise locations [see Figs. 1(b)-1(d) and Sec. S1 in the Supplementary Material for more details]. The amount of gas, and therefore the volume, and the size of microbubbles can be finely tuned by varying the laser power and exposure area during the one-step process (see Secs. S2 and S3 in the Supplementary Material).

The microbubbles can be identified by the observation of Newton's rings that are formed by the interference of light reflected by interfaces between the trapped gas and graphene and between the gas and substrate interface, as indicated in Figs. 1(d) and 1(e). The lateral size of the microbubble is defined by the outermost dark ring that is formed via destructive interference at the edge of the microbubble. The height of the microbubbles can be calculated from the number of rings by a simple equation: $2 h n_{\text {gas }}=m \lambda$, where $n_{\text {gas }}$ is the refractive index of the trapped gas $(\sim 1), \lambda$ is the illumination wavelength (650 nm), and $m$ is an integer corresponding to the number of rings. The highly symmetric Newton's rings observed in Fig. 1(e) and their close agreement with the theoretically calculated pattern in Fig. 1(d) suggest that the produced microbubble has a near-perfect spherical curvature, which is attributed to the exceptional mechanical strength of graphene.

The ability to generate and eliminate microbubbles of various lateral dimensions and heights is demonstrated in Fig. 2. The pristine area before irradiation is shown in Fig. 2(a). As shown in Figs. 2(b)-2(d), the GO film was irradiated in locations indicated by the squares of different sizes. It can be clearly seen that the lateral dimensions and the number of Newton's rings (height of the microbubble) increase with increasing laser exposure area (see Sec. S4 in the Supplementary Material). This is expected since the laser irradiation of a larger area leads to a greater reduction of GO and the release of more gas. The microbubbles can be eliminated by increasing the laser power to ablate the graphene film to release the gases inside the bubbles [Figs. 2(e)-2(h)]. In Fig. 2(h), the surface of the reduced GO film after the removal of microbubbles is shown. Our analysis reveals that the film is largely flat after release of the gases, due to the high elasticity of graphene. The process for eliminating the bubbles takes about $1.5 \mathrm{~s}$ and depends on the volume of enclosed gas and the area of the ablated film. Microbubble generation and gas release can be viewed in Videos $\mathrm{S} 1$ and $\mathrm{S} 2$.

The surface curvature and the volume of microbubbles can be accurately calculated by tracing the position of the rings and reconstructing the surface profile, as shown in Fig. 3. In Fig. 3(a), the area of the microbubble is marked with a white dashed circle and the cross section of the Newton's rings is marked with a yellow dashed line. The measured radial intensity 


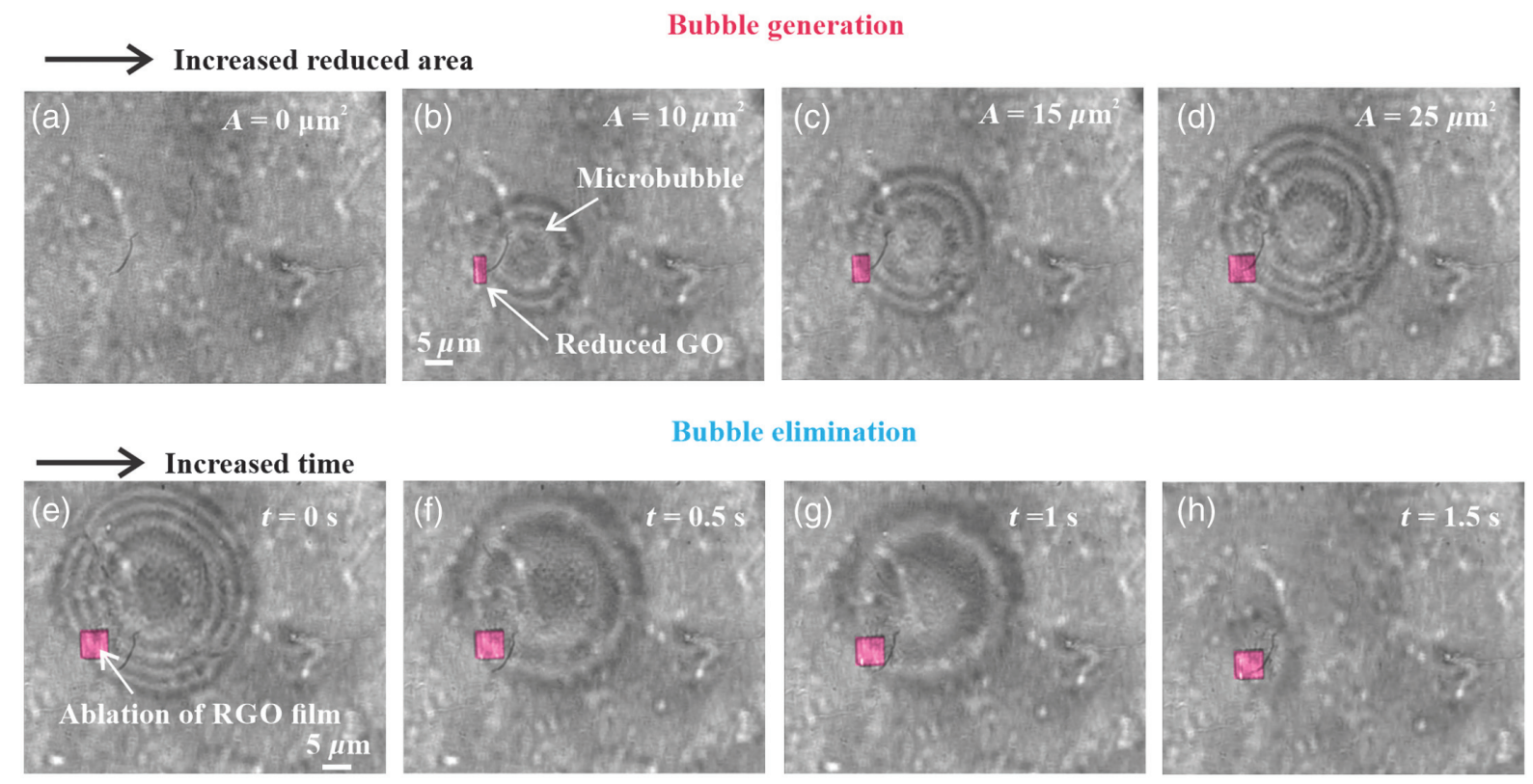

Fig. 2 In situ optical microscopic images showing the process of the microbubble generation and elimination. (a) GO sample before photoreduction and (b)-(d) generated microbubbles corresponding to different reduced areas (Video S1). (e)-(h) Elimination of microbubbles by ablating the reduced area and measured at different times (Video S2). The reduced area is highlighted by the pink color (Video S1, MP4, 1.12 MB [URL: https://doi.org/10.1117/1.AP.2.5.055001.1]; Video S2, MP4, 1.00 MB [URL: https://doi.org/10.1117/1.AP.2.5.055001.2]).

distributions of the rings in different sized microbubbles shown in Fig. 2 are plotted in Fig. 3(b) and appear as a series of bright and dark fringes. The corresponding reconstructed surface curvatures are shown in Fig. 3(c) and indicate that the height of the microbubbles increases with the size of irradiated area.
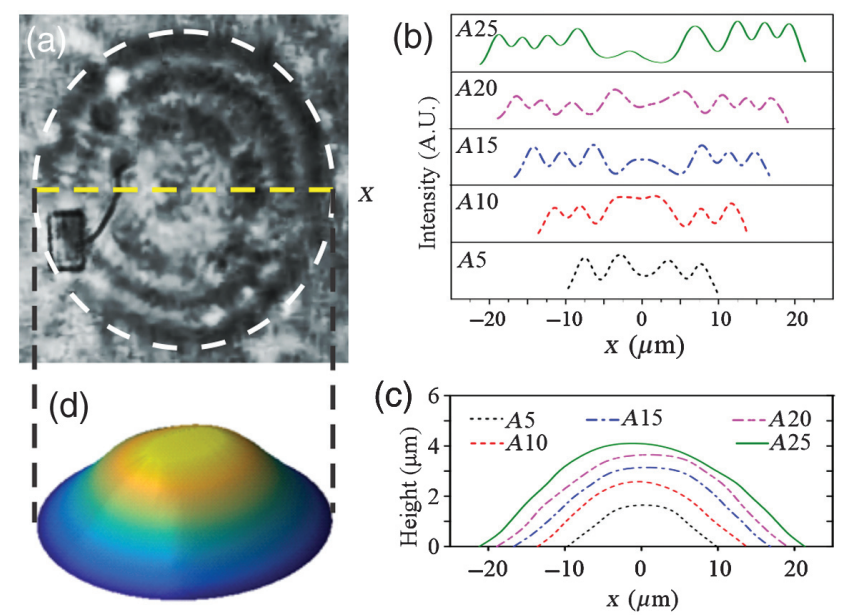

Fig. 3 Bubble characterization. (a) An in situ optical microscopic image of the Newton's rings of the microbubble. The overall area of the microbubble is highlighted by the white circle and the $x$ direction is marked by the yellow dash line. (b) Intensity profiles of the interference pattern of the microbubbles with different reduced areas. (c) Reconstructed surface profiles of the microbubbles with different reduced areas. A5 to A25 correspond to a photoreduced area of 5 to $25 \mu \mathrm{m}^{2}$. (d) Reconstructed 3D profile of the A15 bubble [shown in (a)].
The three-dimensional (3D) profile of a typical microbubble is shown in Fig. 3(d) and reveals that it exhibits a highly symmetric spherical shape. The uniform thickness of the graphene film, which ensures the isotropic surface tension along all directions, is a key parameter for achieving a highly symmetric spherical surface of the bubble. In order to confirm that the near-perfect spherical surface is achieved, we have fitted the surface curvature with a perfect circle function, which shows a good match (Sec. S6 in the Supplementary Material). This means that the surface profile of the microbubbles is very close to an ideal spherical shape.

\section{Results}

The highly uniform surface quality and near-perfect spherical shape of the microbubbles make them ideal reflective microlenses for focusing optical energy. A microscopic imaging system combined with a CCD camera, as shown in Fig. 4(a), was used to measure the intensity distributions in the focal plane of microlenses so that key parameters could be extracted. This was done by placing the graphene microbubble sample on a 1D piezoelectric scanning stage, which scans along the direction of the optical axis ( $z$ direction) to record a layer-by-layer series of $2 \mathrm{D}$ intensity distributions of the focal spot (the characterization process can be viewed in Videos S3 and S4). To evaluate the focal length of the microbubble, the radius $(R)$ of the sphere was calculated from the measured radius $(r)$ and height $(h)$ of the microbubble, as shown in Fig. 4(b). The focal length of the microbubble can then be obtained from $f=R / 2$. The numerical aperture (NA) of the microbubble, a parameter that directly determines the resolution of the bubble focus, can be determined using NA $=r / f$. In addition, the full-width at half-maximum (FWHM) of the focal spot $(d)$ is $d=\lambda /(2 \times \mathrm{NA})$, which can 

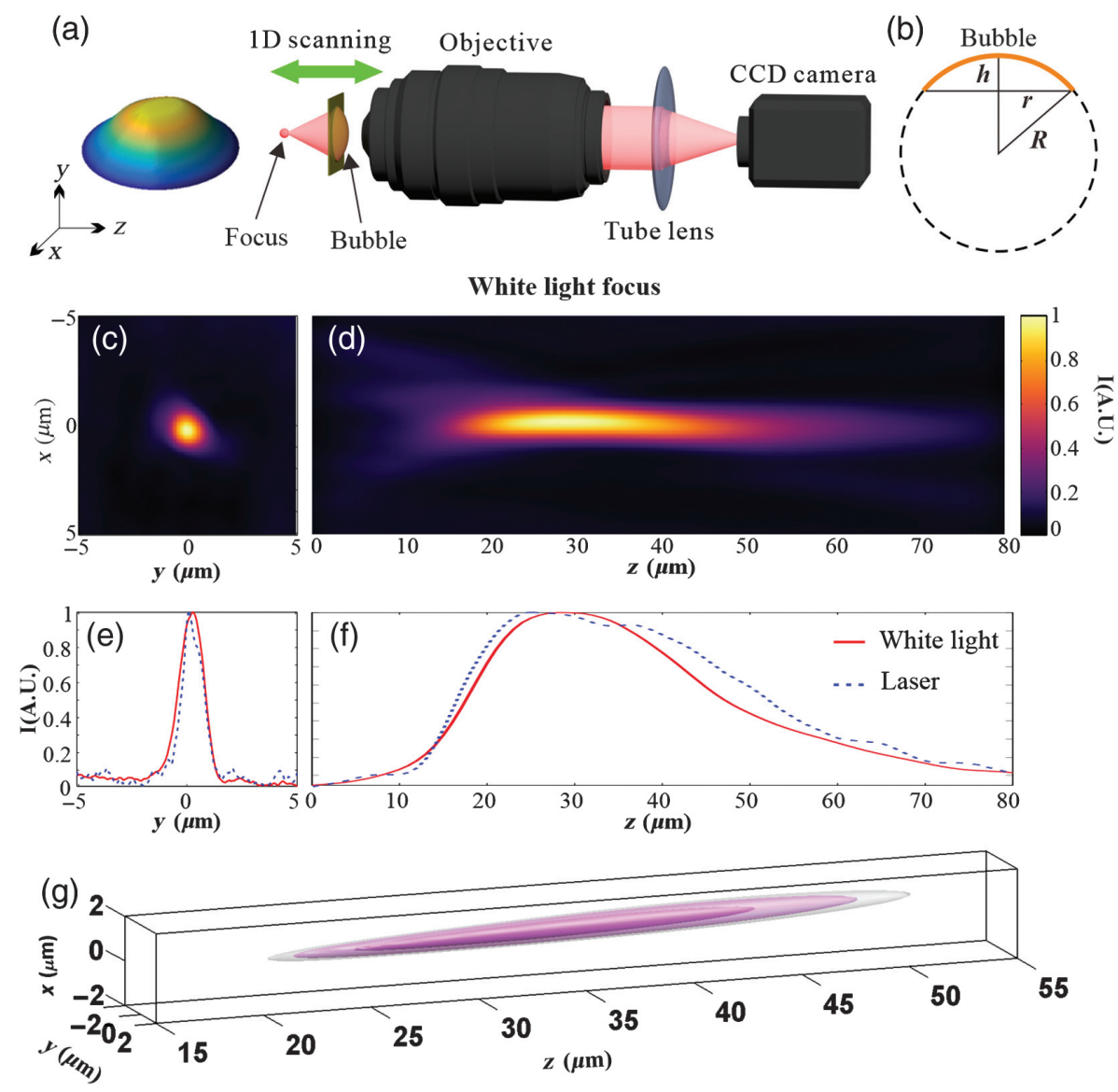

Fig. 4 Focusing photonic jet with a GO microbubble. (a) Schematic of optical setup for characterizing the GO microbubbles. Left: Experimentally reconstructed 3D image of the characterized GO microbubble. (b) Schematic drawing of the shape of the microbubble as part of a sphere. $h$, height of the bubble; $r$, radius of the bubble; and $R$, radius of the entire sphere. Intensity distribution of the focal spot under white light illumination in the (c) $x-y$ plane and (d) $x-z$ plane (Video S3); the intensity plots along the (e) $y$ direction and (f) $z$ direction (Videos S3 and S4). (g) The surface plot of the experimentally measured $3 \mathrm{D}$ focal spot. The contours from inside correspond to the intensities of 0.8, 0.6, and 0.5 of the normalized peak intensity (Video S3, MP4 1.06 MB [URL: https:// doi.org/10.1117/1.AP.2.5.055001.3]; Video S4, MP4, 606 KB [URL: https://doi.org/10.1117/ 1.AP.2.5.055001.4]).

be used to characterize the focusing performance of the microlenses. The focal intensity distribution of the microbubble illuminated with a He-Ne laser $(633 \mathrm{~nm})$ shows a surprisingly strong 3D focal spot (see Sec. S9 and Fig. S8 in the Supplementary Material), indicating the high quality of the microbubble. Furthermore, from the measured radius and height of the microbubble, NA is estimated to be 0.27 , which corresponds to $d=$ $1.18 \mu \mathrm{m}$, matching well with the measured FWHM in the $y$ direction. This demonstrates that diffraction-limited focusing can be achieved by the graphene microbubble.

Compared with diffractive graphene flat lenses, ${ }^{21-24}$ a key advantage of reflective graphene microlenses is that the reflection occurs at the gas-reduced GO interface and therefore material dispersion does not play a role. This means that graphene microbubble microlenses are able to focus light at different wavelengths without dispersion, similar to the reflection lenses in Fourier transform infrared spectrometers. ${ }^{25}$ To verify this unique feature experimentally, an ultrabroadband white light source (a halogen lamp) was employed for focusing measurements.
The resulting intensity distributions in the $x-y$ plane (focal plane) and the $x-z$ plane are shown in Figs. 4(c) and 4(d), respectively, and can be seen to exhibit photonic jet-like features. The corresponding intensity plots along the $y$ direction and $z$ direction are shown in Figs. 4(e) and 4(f). Negligible broadening of the FWHM $(\sim 1.31 \mu \mathrm{m})$ is observed along the $y$ direction despite the incoherence of the light source. In contrast, the FWHM along the $z$ direction is significantly reduced to $30 \mu \mathrm{m}$ due to the broad spectral distribution of the light source, which increases the resolution. These results demonstrate that a 3D focal spot can be achieved with ultra-broadband white light illumination, as shown in Fig. 4(g). The focal spots of different wavelengths overlap extremely well with each other at all normalized intensity levels $(0.8,0.6$, and 0.5$)$, as indicated by different colors [Fig. $4(\mathrm{~g})]$.

\section{Discussion}

This microbubble photonic jet created by reflective graphene microlenses is technologically significant and has clear advantages 
over a photonic jet that is generated by transmission through a glass microsphere. ${ }^{26}$ The reflection mode operation of our microlenses means that they are insensitive to material dispersion ${ }^{27}$ and therefore can generate diffraction-limited focus for broad wavelengths. Second, the size and radius of the microbubbles are readily tunable in situ, allowing adjustable focusing properties. Therefore, GO microbubbles offer a flexible and in situ tuning mechanism for focal length without degrading the focal resolution of the photonic jet. The large focal length leads to long working distances, which is highly desirable for 3D biological imaging in lab-on-a-chip devices. ${ }^{28}$ As a result, graphene microbubbles are expected to find broad applications in integrated devices for imaging, spectroscopy, and sensing by generating microlenses at arbitrarily desired positions directly on substrates.

\section{Acknowledgments}

Baohua Jia thanks the Australian Research Council for its support (Nos. DP150102972, DP190103186, and IC180100005). Minghui Hong thanks the National Research Foundation of Singapore for its support (No. NRF-RP15-2015-04). The authors thank David Mesa Saldarriaga for his artistic input in the figure presentation. The authors claim no conflicts of interests in this work. Dan Li acknowledges the financial support from the Australian Research Council (No. FL180100029).

\section{References}

1. R. Dijkink and C.-D. Ohl, "Laser-induced cavitation based micropump," Lab Chip 8(10), 1676-1681 (2008).

2. P. Garstecki et al., "Mixing with bubbles: a practical technology for use with portable microfluidic devices," Lab Chip 6(2), 207-212 (2006).

3. M. Lee et al., "Stabilization and fabrication of microbubbles: applications for medical purposes and functional materials," Soft Matter 11(11), 2067-2079 (2015).

4. M. Prakash and N. Gershenfeld, "Microfluidic bubble logic," Science 315(5813), 832-835 (2007).

5. P. Prentice et al., "Membrane disruption by optically controlled microbubble cavitation," Nat. Phys. 1(2), 107-110 (2005).

6. S. S. Dastgheyb and J. R. Eisenbrey, "Microbubble applications in biomedicine," in Handbook of Polymer Applications in Medicine and Medical Devices, K. Modjarrad and S. Ebnesajjad, Eds., pp. 253-277, William Andrew Books, Elsevier B.V., Amsterdam (2014).

7. T. Okamoto, T. Suzuki, and N. Yamamoto, "Microarray fabrication with covalent attachment of DNA using bubble jet technology," Nat. Biotechnol. 18(4), 438-441 (2000).

8. L. Lin et al., "Bubble-pen lithography," Nano Lett. 16(1), 701-708 (2016).

9. M. Sumetsky, Y. Dulashko, and R. Windeler, "Optical microbubble resonator," Opt. Lett. 35(7), 898-900 (2010).

10. X. Zhang and D. Lohse, "Perspectives on surface nanobubbles," Biomicrofluidics 8(4), 041301 (2014).

11. J. Zabel et al., "Raman spectroscopy of graphene and bilayer under biaxial strain: bubbles and balloons," Nano Lett. 12(2), 617-621 (2012).

12. T. Georgiou et al., "Graphene bubbles with controllable curvature," Appl. Phys. Lett. 99(9), 093103 (2011).

13. Y. Yang et al., "Graphene-based multilayered metamaterials with phototunable architecture for on-chip photonic devices," ACS Photonics 6(4), 1033-1040 (2019).

14. H. Lin et al., "A 90-nm-thick graphene metamaterial for strong and extremely broadband absorption of unpolarized light," Nat. Photonics 13(4), 270-276 (2019).
15. K.-T. Lin et al., "Structured graphene metamaterial selective absorbers for high efficiency and omnidirectional solar thermal energy conversion," Nat. Commun. 11, 1389 (2020).

16. S. K. Sundaram and E. Mazur, "Inducing and probing non-thermal transitions in semiconductors using femtosecond laser pulses," Nat. Mater. 1(4), 217-224 (2002).

17. X. Zheng et al., "Laser trimming of graphene oxide for functional photonic applications," J. Phys. D Appl. Phys. 50(7), 074003 (2017).

18. Y. L. Zhang et al., "Photoreduction of graphene oxides: methods, properties, and applications," Adv. Opt. Mater. 2(1), 10-28 (2014).

19. H. Zhang et al., "Ultrahigh heating rate induced micro-explosive production of graphene for energy storage," J. Power Sour. 442(1), 227224 (2019).

20. T. Yang et al., "Tailoring pores in graphene-based materials: from generation to applications," J. Mater. Chem. A 5(32), 16537-16558 (2017).

21. X. Zheng et al., "Highly efficient and ultra-broadband graphene oxide ultrathin lenses with three-dimensional subwavelength focusing," Nat. Commun. 6, 8433 (2015).

22. G. Cao et al., "Resilient graphene ultrathin flat lens in aerospace, chemical, and biological harsh environment," ACS Appl. Mater. Interfaces 11(22), 20298-20303 (2019).

23. G. Cao et al., "An accurate design of graphene oxide ultrathin flat lens based on Rayleigh-Sommerfeld theory," Opto-Electron. Adv. 1(7), 180012 (2018).

24. X. Li et al., "Graphene metalens for particle nanotracking," Photonics Res. 8(8), 1316-1322 (2020).

25. J. Li, B. Jia, and M. Gu, "Engineering stop gaps of inorganicorganic polymeric 3D woodpile photonic crystals with post-thermal treatment," Opt. Express 16(24), 20073-20080 (2008).

26. A. Heifetz et al., "Photonic nanojets," J. Comput. Theor. Nanosci. 6(9), 1979-1992 (2009).

27. B. Born et al., "Integration of photonic nanojets and semiconductor nanoparticles for enhanced all-optical switching," Nat. Commun. 6 , 8097 (2015).

28. F. Qin et al., "Shaping a subwavelength needle with ultra-long focal length by focusing azimuthally polarized light," Sci. Rep. 5(6), 9977 (2015).

Han Lin received his BS and MS degrees in physics from Xiamen University in 2005 and 2008, respectively, and his $\mathrm{PhD}$ in optics from Swinburne University of Technology in 2013. He is a senior research fellow at Swinburne University of Technology, Australia. He is the author of more than 50 journal papers and has written 1 book. His current research interests include nanophotonics and 2D materials, and laser nanofabrication. He is a member of SPIE.

Scott Fraser received his BS degree in engineering from Swinburne University of Technology, Australia, in 2015. He is a PhD student at Swinburne University of Technology. His current research interests include nanophotonics and 2D materials, and laser nanofabrication. He is a member of SPIE.

Minghui Hong is the director of the Optical Science and Engineering Center at NUS. He specializes in laser microprocessing and nanofabrication, and optical engineering and applications. He has co-authored 10 book chapters, 24 patents granted, and 400+ scientific papers in Nature, Chemical Reviews, Nature Nanotechnology, Advanced Materials, Nature Communications, Nano Letters, ACS Nano, Laser and Photonics Reviews, Light: Science and Applications, etc., and 50+ plenary/keynote/invited talks in international conferences. He is also a fellow of the Optical Society of America, a fellow of the International Society for Optics and Photonics, and a founding fellow of the International Academy of Photonics and Laser Engineering.

Manish Chhowalla is the Goldsmiths' Professor of materials science. His research interests are in the fundamental studies of atomically thin two- 
dimensional transition metal dichalcogenides (TMDs). In particular, his group studies the optical and electronic properties of different phases of 2D-TMDs. He has demonstrated that it is possible to induce phase transformations in atomically thin materials and utilize phases with disparate properties for field effect transistors, catalysis, and energy storage.

Dan Li is an Australian Laureate Fellow and professor in materials science and engineering in the Department of Chemical Engineering at the University of Melbourne, Australia. His current research interests include synthesis of graphene and 2D nanomaterial-based soft materials and their applications in nanoionics, molecular/ion separation, electrochemical energy storage and conversion, and biomedicine.
Baohua Jia received her $\mathrm{PhD}$ in 2007 from Swinburne University of Technology, Australia. She is a full professor and a founding director of the Centre for Translational Atomaterials and Research Leader at Swinburne University of Technology. Her research focuses on the fundamental light and nanomaterial interaction. In particular, her work on laser manipulation of two-dimensional materials has led to the design and fabrication of functional nanostructures and nanomaterials for effective harnessing and storage of clean energy from sunlight, purifying water and air for clean environment and imaging and spectroscopy, and nanofabrication using ultrafast laser toward fast-speed all-optical communications and intelligent manufacturing. 